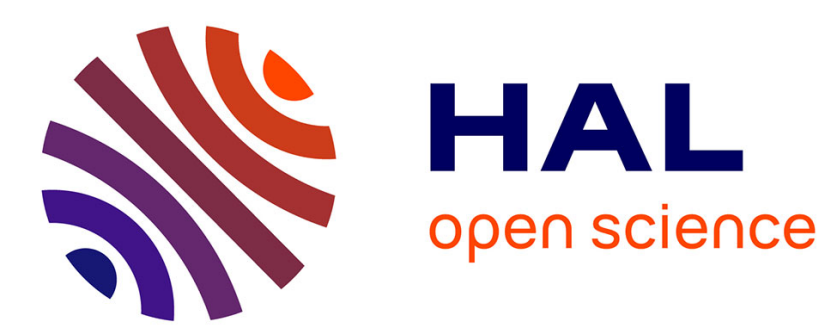

\title{
Theoretical approach in real space to the masses of protons and neutrons
}

Nathalie Olivi-Tran

\section{To cite this version:}

Nathalie Olivi-Tran. Theoretical approach in real space to the masses of protons and neutrons. Nuclear and Particle Physics Proceedings, 2021, 312-317C, pp.78-81. 10.1016/j.nuclphysbps.2021.05.021 . hal-03234765

\section{HAL Id: hal-03234765 \\ https://hal.science/hal-03234765}

Submitted on 25 May 2021

HAL is a multi-disciplinary open access archive for the deposit and dissemination of scientific research documents, whether they are published or not. The documents may come from teaching and research institutions in France or abroad, or from public or private research centers.
L'archive ouverte pluridisciplinaire HAL, est destinée au dépôt et à la diffusion de documents scientifiques de niveau recherche, publiés ou non, émanant des établissements d'enseignement et de recherche français ou étrangers, des laboratoires publics ou privés. 


\title{
Theoretical approach in real space to the masses of protons and neutrons*
}

\author{
N.Olivi-Tran ${ }^{1, a}$ \\ ${ }^{a}$ Laboratoire Charles Coulomb, C.N.R.S., Universite de Montpellier, place Eugene Bataillon, 34095 Montpellier cedex 5, France
}

\begin{abstract}
We make the hypothesis that our universe is threedimensional and curved, hence our universe may be embedded in a fourdimensional Euclidean space where the four dimensions are $(x, y, z, t)$. The fourth dimension is time $t$ which is treated like a spatial dimension. Straightforwardly, this Euclidean space has an underlying hypersquare array for which the edges have a width of one Planck length. The eigenfunctions of each edge of this array are $\sqrt{2} \exp \left(i x_{i}\right)$ where $x_{i}=x, y, z$ or $t$. As previously published, the quarks are threedimensional in real space. The quark up has a mass of $2^{21} \mathrm{eV} / \mathrm{c}^{2}$ and the quark down $2^{22} \mathrm{eV} / \mathrm{c}^{2}$ (the quark down is an excited state of the quark up). Because the quark up is threedimensional (like the apex of a tetrahedron), each edge of the quark has an eigenvalue of $2^{7} \mathrm{eV} / \mathrm{c}^{2}$. Let us consider that the color charge of the gluons are in fact the coordinates in real space ( $x$ : red; $y:$ blue; $z$ : green). The gluons have no mass so they have no temporal dimension. Each pair of quarks, share one gluon (the gluons interfere with the quarks edges).Gluons are bosons and quarks are fermions so for each edge of the underlying array (except the temporal edges) we calculate the ground states. In one proton or in one neutron, there are 3 gluons and 3 quarks; as only the temporal edges of the 3 quarks are not in the ground states and because the protons and neutrons obey the Schrödinger equation, the masses of the proton or neutron are equal to $2^{30} / \ln (\pi) e V / c^{2}=937 \mathrm{MeV} / \mathrm{c}^{2}$. The difference between the masses of the proton and neutron comes from the difference between the experimental masses and the theoretical masses of quarks up and down. Straightforwardly, we found theoretical values of the masses of protons and neutrons within $2 \%$ of the experimental values of the masses of protons and neutrons.
\end{abstract}

Keywords: real space theory, theoretical mass of protons, theoretical mass of neutrons

\section{Introduction}

The masses of protons and neutrons remained a long time without a theoretical explanation to their value. Protons contain 2 quarks up and 1 quark down, while neutrons contain 1 quark up and 2 quarks down. There are also 3 gluons within protons and neutrons, these gluons carry the strong force. The experimental mass of protons is equal to $938.2720 \mathrm{MeV} / \mathrm{c}^{2}$, while the experimental mass of neutrons is equal to $939.565 \mathrm{MeV} / \mathrm{c}^{2}$.

*Talk given at 23rd International Conference in Quantum Chromodynamics (QCD 20), 27 october-30 October 2020, Montpellier FR

Email address: nathalie.olivi-tran@umontpellier.fr (N.Olivi-Tran ${ }^{1,}$ )

${ }^{1}$ Speaker, Corresponding author.
Indeed, knowing the masses of quarks up and down and also knowing that the gluons have no mass, the mass of a proton (resp. neutron) cannot be explained by simply adding the masses of quarks up and down.

In order to give a theoretical explanation to the masses of protons and neutrons, I will use the same approach in real space than the calculation of the masses of the 3 families of elementary fermions [1-3].

I make the assumption that our threedimensional universe is embedded in a four dimensional euclidean space. Time is a function of the fourth dimension of this space [4-6]. Moreover,the following items have been found in previous articles [1-3] and will be used to calculate the masses of protons and neutrons:

- Calculation of particles masses in the $(\mathrm{x}, \mathrm{y}, \mathrm{z}, \mathrm{t})$ spacetime 


\begin{tabular}{|c|c|c|}
\hline quark & Theoretical mass $\left(\mathrm{eV} / \mathrm{c}^{2}\right)[1-3]$ & Experimental mass $\left(\mathrm{eV} / \mathrm{c}^{2}\right)[7]$ \\
\hline up & $2^{21}=2.09 \mathrm{Mev} / \mathrm{c}^{2}$ & $2.2 \mathrm{MeV} / \mathrm{c}^{2}$ \\
down & $2^{21} .2^{2} / 2=4.19 \mathrm{MeV} / \mathrm{c}^{2}$ & $4.7 \mathrm{MeV} / \mathrm{c}^{2}$ \\
\hline
\end{tabular}

Table 1: Theoretical and experimental masses of the quarks up and down.

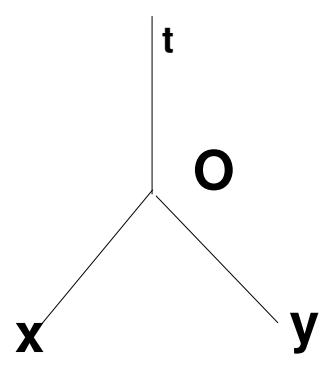

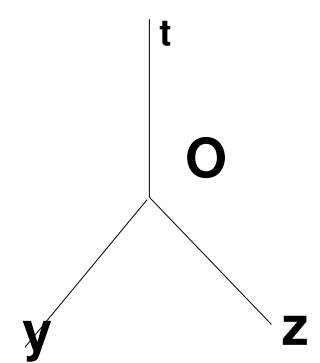

QUARKS

Figure 1: Geometry of quarks

- Time is equivalent to a space dimension (except that it is not possible to go backwards in time)

- Spacetime has an underlying hypersquare array of edge length $\hbar$

- Eigenfunctions ( in $(x, y, z, t)$ space) of this hypersquare array (reduced parameters) are $\sqrt{2} \exp (i x)$ for space, $\sqrt{2} \exp (i t)$ for time (the eigenvalues of these eigenfunctions are equal to $\sqrt{2}$ in the ground states)

- Eigenvalues for excited states of these eigenfunctions equal to $p^{2} / 2$ (with $p$ an integer number)

With these items, we found the masses of the first family of elementary fermions which are equal to integer powers of 2 (in $e V / c^{2}$ ) [1-3]. The masses of elementary fermions which belong to the 2 nd and 3rd family of fermions are excited states of the elementary fermions of the first family; thus these particles masses are multiplied by $p^{2} / 2$ with respect to the masses of the first family of elementary fermions (with $p$ an integer) [13].

In the following section, I will present the hypotheses for quarks, gluons and protons and neutrons. This leads to the theoretical value of the mass of protons which equals $937 \mathrm{MeV} / \mathrm{c}^{2}$. The theoretical value of the mass of neutrons is found to be less than $2 \%$ larger than the experimental value.

\section{Hypotheses}

\subsection{Quarks}

I present here the hypotheses for quarks.

- quarks up and down are 3d [8]

- quarks are fermions

- quarks obey the Dirac equation

- the color charge of one quark for example $(t, x, y)$ is blue, $(t, x, z)$ is green and $(t, y, z)$ is red ( $x$ : red; $y$ :green $; z$ : blue)

Because the quark up is threedimensional (like the apex of a tetrahedron, see figure 1), each edge of the quark has an eigenvalue of $2^{7} \mathrm{eV} / \mathrm{c}^{2}$ indeed the mass of the quark up is equal to $2^{21} \mathrm{eV} / \mathrm{c}^{2}$ (see table 1). The experimental masses of quarks up and quarks down are larger than the theoretically found values of their masses (see also table 1). This will explain the mass difference between protons and neutrons.

\subsection{Gluons}

I present here the hypotheses for gluons which carry the strong force within protons and neutrons nuclei.

- Gluons are $2 \mathrm{~d}$

- The gluons have no mass so they have no temporal dimension

- gluons are bosons 


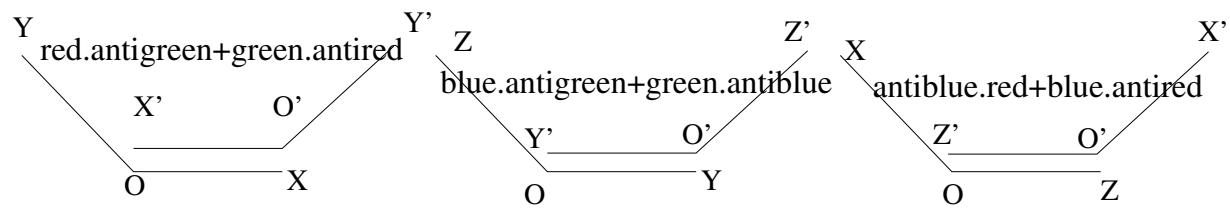

Figure 2: Geometry of gluons

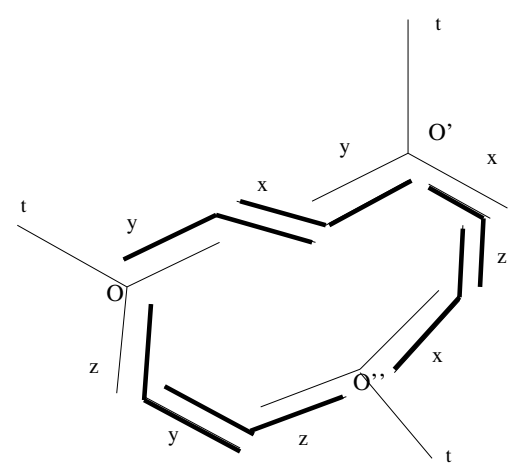

Figure 3: Geometry of protons or neutrons: thin lines represent quarks and bold lines represent gluons

- gluons obey the Klein-Gordon equation

- The color charge of gluons are in fact the coordinates in real space ( $x$ : red; $y$ :green ; $z$ : blue); see figure 2. For the coordinate $x$, red corresponds to a vector $\mathbf{O X}$ and antired to its opposite, the vector $\mathbf{X}^{\prime} \mathbf{O}^{\prime}$. For coordinates $y$ and $z$ respectively, the same applies with $\mathbf{O Y}$ (resp. $\mathbf{Y}^{\prime} \mathbf{O}^{\prime}$ ) and $\mathbf{O Z}$ (resp. $\left.\mathbf{Z}^{\prime} \mathbf{O}^{\prime}\right)$.

\subsection{Protons and neutrons}

Protons and neutrons obey the Schrödinger equation. The Schrödinger equation writes [9]:

$$
\frac{\hbar^{2}}{2 m} \nabla^{2} \psi(x, t=0)=\frac{\hbar}{\omega} \frac{\partial^{2}}{\partial t^{2}} \psi(x=0, t)
$$

this leads to

$$
\pi \psi(x, t=0)=\psi(x=0, t)
$$

(in normalized units $m=1, \hbar=1, \omega=2 \pi v$ with the frequency $v=1$ ). This leads to

$$
\pi \exp (i x)=\exp (i t)
$$

and finally to

$$
x=t / \ln (\pi)
$$

This last equation gives the relation between time $t$ and spatial dimensions (for example $x$ ) when calculating the masses of protons and neutrons.
This relation is not valid for quarks as these last do not obey the Schrödinger equation but the Dirac equation. Because gluons have no mass, this relation is not valid when calculating the eigenvalues of gluons.

\section{Calculations of the masses of protons and neu- trons}

In one proton or in one neutron, there are 3 gluons and 3 quarks. Each pair of quarks share one gluon (the gluons interfere with the quarks edges), see figure 3 . The quarks are represented by thin lines and gluons by bold lines.

Gluons are bosons and quarks are fermions so each edge of the underlying array (except the temporal edges) is in the ground state because quarks and gluons interfere on these edges. The eigenvalue of each spatial edge of the quarks is equal to $\sqrt{2} e V / c^{2}$. There are 6 spatial edges of the quarks in one proton or one neutron (there are 6 thin segments within quarks with a spatial dimension: see figure 3 ). This gives a spatial eigenvalue equal to $\sqrt{2}^{6}=2^{3} \mathrm{eV} / \mathrm{c}^{2}$ for the 3 quarks. The eigenvalue of each edge of gluons is also equal to $\sqrt{2}$ (each bold segment, within gluons, has an eigenvalue equal to $\sqrt{2}$, see figure 3 ). Because there are 4 edges within one gluon, each gluon has an eigenvalue equal to $\sqrt{2^{4}}=2^{2} \mathrm{eV} / \mathrm{c}^{2}$. There are 3 gluons in one proton or one neutron so the eigenvalue for the 3 gluons is equal to $2^{6} \mathrm{eV} / \mathrm{c}^{2}$. 
Only the temporal edges of the 3 quarks are not in the ground states, moreover the temporal eigenvalues are equal to $\left(2^{7}\right)^{3} / \ln (\pi)$ (see section 2.1 and equation 4) because there are 3 temporal edges of the quarks: 3 thin segments in figure 3 . So the masses of the proton or neutron are equal to $\left(2^{7}\right)^{3} \cdot\left(2^{6}\right) \cdot\left(2^{3}\right) / \ln (\pi)=$ $2^{30} / \ln (\pi) e V / c^{2}=937 M e V / c^{2}$ (we multiply all the eigenvalues of quarks and gluons). This value is close to the experimental values of the masses of protons and neutrons: $938.2720 \mathrm{MeV} / \mathrm{c}^{2}$ for protons and $939.565 \mathrm{MeV} / \mathrm{c}^{2}$ for neutrons.

Theoretically, protons and neutrons have the same mass. However, , the experimental mass of quarks up is equal to $2.2 \mathrm{MeV} / \mathrm{c}^{2}$ and the experimental mass of quarks down is equal to $4.7 \mathrm{MeV} / \mathrm{c}^{2}$ (see table 1). So, the experimental temporal edge for the quark up has an eigenvalue equal to $\left(2.2 .10^{6}\right)^{1 / 3}=130.059 \mathrm{eV} / \mathrm{c}^{2}$ and the experimental temporal edge for the quark down has an eigenvalue equal to $\left(4.7 .10^{6} / 2\right)^{1 / 3}=132.95 \mathrm{eV} / \mathrm{c}^{2}$. So the mass ratio between protons ( 2 quarks up and 1 quark down) and neutrons (1 quark up and 2 quarks down) is equal to $130.059 / 132.95=0.978 \%$.

\section{Conclusion}

We found theoretically the value of the mass of the proton which is equal to $937 \mathrm{MeV} / \mathrm{c}^{2}$. This value equals the experimental value within $0.1 \%$. The theoretical value of the mass of the neutron is included between $937 \mathrm{MeV} / \mathrm{c}^{2}$ and $937 / 0.978=958 \mathrm{MeV} / \mathrm{c}^{2}$ which equals the experimental mass within $1.02 \%$.

\section{References}

[1] N.Olivi-Tran,The masses of the first family of fermions and of the Higgs boson are equal to integer powers of 2 Nuclear and Particle Physics Proceedings (2015) vol.258-259C pp. 272-275

[2] N.Olivi-Tran Theoretical approach to the masses of the elementary fermions Nuclear and Particle Physics Proceedings 309-311C (2020) pp. 73-76

[3] N.Olivi-Tran Theoretical Calculations of the Masses of the Elementary Fermions book chapter in Accelerators and Colliders 2020 (Intechopen)

[4] N.Olivi-Tran and P.M.Gauthier, The FLRW cosmological model revisited: Relation on the local time with the local curvature and consequences on the Heisenberg uncertainty principle Adv. Studies Theor. Phys. (2008), vol.2, no 6, 267-270

[5] N.Olivi-Tran What if our three dimensional curved universe was embedded in four dimensional space? Consequences on the EPR paradox Adv. Studies Theor. Phys.,(2009), Vol. 3, no. $12,489-492$

[6] N.Olivi-Tran Dimensional analysis of Einstein's fields equations Adv. Studies Theor. Phys.(2009), Vol. 3, no. 1, 9 - 12

[7] C. Patrignani et al. (Particle Data Group), Review of Particle Physics Chinese Physics C,(2016) vol. 40, 100001
[8] N.Olivi-Tran and N.Gottiniaux A classification of elementary particles in $d=4$ following a simple geometrical hypothesis in real space Adv. Studies Theor. Phys., (2013), Vol. 7, no. 18, 853-857

[9] N.Olivi-Tran, Is time quantized? Advanced Studies in Theoretical Physics Vol. 6, 2012, no. 12, 563 - 568 (open access journal) 